\title{
FRACTIONAL BROWNIAN MOTION: THEORY AND APPLICATIONS
}

\author{
LAURENT DECREUSEFOND AND ALI SULEYMAN ÜSTÜNEL
}

\begin{abstract}
We present new theoretical results on the fractional Brownian motion, including different definitions (and their relationships) of the stochastic integral with respect to this process, Girsanov theorem, Clark representation formula, Itô formula and so on. Several applications (such as non-linear filtering theory, queuing networks, mathematical finance) are given as applied examples of these theoretical results.
\end{abstract}

RÉSUMÉ. Nous présentons de nombreux et récents résultats théoriques concernant le mouvement brownien fractionnaire. En particulier, nous rappelons ce que sont le théorème de Girsanov, la formule de représentation de Clark, la formule d'Itô, etc. Ces résultats sont appliqués dans de nombreux domaines tels que la théorie du filtrage non-linéaire, les files d'attente, la finance.

\section{INTRODUCTION}

The theoretical study of the fractional Brownian motion was originally motivated by new problems in mathematical finance and telecommunication networks. In engineering applications of probability, stochastic processes are often used to model the input of a system. For instance, the mathematical finance requires stochastic models for the time evolution of assets and the queuing networks analysis is based on models of the offered traffic. Hitherto, the stochastic processes used in these fields are often supposed to be Markovian. However, recent studies [18] show that real inputs exhibit long-range dependence : the behavior of a real process after a given time $t$ does not only depend on the situation at time $t$ but also of the whole history of the process up to time $t$. Moreover, it turns out that this property is far from being negligible because of the effects it induces on the expected behavior of the global system [26].

Another property that have the processes encountered in applications (at least in communication networks) is the self-similarity (see [18]): their behavior is stochastically the same, up to a space-scaling, whatever the time-scale is - that is to say that the process $\left\{X_{\alpha t}, t \in[0,1]\right\}$ has the same law as the process $\left\{\alpha^{H} X_{t}, t \in[0,1]\right\}$, where $H$ is called the Hurst parameter. For an historical account on the works of Hurst, see [21]. Several estimations on real data tend to show that $H$ often lies between 0.7 and 0.8 whereas for instance, the usual Brownian motion has a Hurst parameter equal to 0.5 but it is also clear that some real processes have a Hurst parameter less than 0.5 - see [6]. There exists several stochastic processes which are self-similar and exhibiting long-range dependence but the fractional Brownian motion (fBm for short) seems to be one of the simplest.

Definition 1.1. For any $H$ in $(0,1)$, the fractional Brownian motion of index (Hurst parameter) $H,\left\{W_{t}^{H} ; t \in[0,1]\right\}$ is the centered Gaussian process whose

\footnotetext{
(C) Société de Mathématiques Appliquées et Industrielles. Typeset by $\mathrm{LT}_{\mathrm{E}} \mathrm{X}$.

L. Decreusefond, A. S. Ustünel: ENST, Dept. INFRES. 46, rue Barrault, 75634 Paris Cedex 13, France. E-mail: decreuse@res.enst.fr ustunel@res.enst.fr.
} 
covariance kernel is given by

$$
R_{H}(s, t)=\mathbf{E}\left[W_{s}^{H} W_{t}^{H}\right] \stackrel{\text { def }}{=} \frac{V_{H}}{2}\left(s^{2 H}+t^{2 H}-|t-s|^{2 H}\right)
$$

where

$$
V_{H} \stackrel{\text { def }}{=} \frac{\Gamma(2-2 H) \cos (\pi H)}{\pi H(1-2 H)} .
$$

This paper aims to give a few aspects of the recent theory and applications of the fractional Brownian motion. We begin by the construction of the process for which recent theoretical advances simplify the computer simulation of samplepaths. Section 4 is devoted to the problem of the definition of a stochastic integral with respect to the fractional Brownian motion. In Section 5, we give several applications in different fields : queuing networks, filtering theory, mathematical finance. The appendix contains a very brief summary of the notion of deterministic fractional calculus we repeatedly use here. Throughout this paper, we will try to show how infinite-dimensional processes arise naturally when studying long-range dependent processes. Figuratively, this amounts to say that we put the memory into the state of the process.

\section{Construction}

We assume now that $H$ is given and fixed. Note that in practical situations, the first task is to determine $H$, there exists a large literature on this subject (see for instance $[23,29]$ and references therein).

\subsection{TheORETICAL APPROACH}

Since $R_{H}$ is a positive definite operator, the Bochner-Minlos theorem ensures that, for any value of $H \in[0,1]$, there exits a unique probability measure on $\mathcal{C}_{0}([0,1] ; \mathbf{R})$ such that the canonical process is a fractional Brownian motion. In particular, for $H=0, W_{t}^{0}=Y$ where $Y$ is a centered Gaussian random variable with variance 1 , for $H=1, W_{t}^{1}=Y . t$ and for $H=1 / 2, W^{1 / 2}$ is the standard Brownian motion.

Beyond this formal result of existence, there exist several explicit representations : in their pioneering work on the subject, Mandelbrot and Van Ness [21], shown that if $B$ is a standard Brownian motion on $\mathbf{R}$, then for $H>1 / 2$,

$$
W_{t}^{H}=\int_{0}^{t}(t-s)^{H-1 / 2} d B_{s}+\int_{-\infty}^{0}\left((t-s)^{H-1 / 2}-|s|^{H-1 / 2}\right) d B_{s}
$$

is a $\mathrm{fBm}(\mathrm{H})$. In a recent paper [11], the authors proved that given any probability space on which lives a $\mathrm{fBm}(\mathrm{H})$, there exits a standard Brownian motion $B$ such that

$$
W_{t}^{H}=\int_{0}^{t} K_{H}(t, s) d B_{s}
$$

where

$$
K_{H}(t, r)=\frac{(t-r)^{H-\frac{1}{2}}}{\Gamma\left(H+\frac{1}{2}\right)} F\left(\frac{1}{2}-H, H-\frac{1}{2}, H+\frac{1}{2}, 1-\frac{t}{r}\right) 1_{[0, t)}(r) .
$$

The Gauss hyper-geometric function $F(\alpha, \beta, \gamma, z)$ (see [24]) is the analytic continuation on $\mathbb{C} \times \mathbb{C} \times \mathbb{C} \backslash\{-1,-2, \ldots\} \times\{z \in \mathbb{C}, A r g|1-z|<\pi\}$ of the power series

$$
\sum_{k=0}^{+\infty} \frac{(\alpha)_{k}(\beta)_{k}}{(\gamma)_{k} k !} z^{k}
$$

ESAIM: Proc., Vol. 5, 1998, 75-86 
Here $(\alpha)_{k}$ denotes the Pochhammer symbol defined by

$$
(a)_{0}=1 \text { and }(a)_{k} \stackrel{\text { def }}{=} \frac{\Gamma(a+k)}{\Gamma(a)}=a(a+1) \ldots(a+k-1) .
$$

It is helpful for computations to note that for $H \leq 1 / 2$,

$$
\tilde{K}_{H}(t, s)=K_{H}(t, s) s^{|H-1 / 2|}(t-s)^{|H-1 / 2|}
$$

and for $H \geq 1 / 2$,

$$
\tilde{K}_{H}(t, s)=K_{H}(t, s) s^{|H-1 / 2|}(t-s)^{-|H-1 / 2|}
$$

are positive, continuous with respect to $(t, s)$, functions - see figure 1 . Moreover, we will use later that for $H>1 / 2$ and $s$ fixed, the map $t \mapsto K_{H}(t, s)$ belongs to $\mathcal{I}_{H-1 / 2, \infty}$.
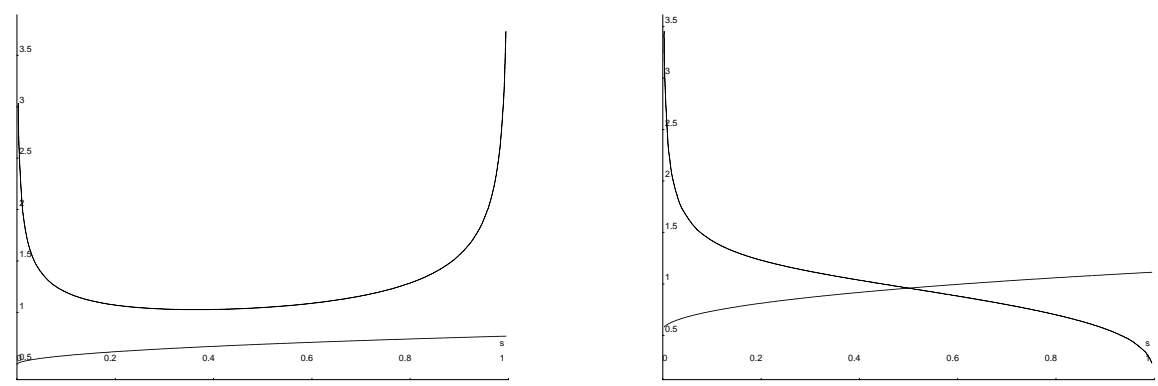

Figure 1. Graph of $K_{H}(1,$.$) and \tilde{K}_{H}(1,$.$) for H=0.2, H=0.8$.

Extending the ideas of Audounet and Montseny [22] to the stochastic situation, Coutin, Carmona and Montseny [4] showed that for $H<1 / 2, W^{H}$ can be written

$$
W_{t}^{H}=\int_{-\infty}^{\infty} X(\xi, t) d \xi
$$

where for any $t$,

$$
\begin{aligned}
X(\xi, t)=2(2 \pi)^{1-2 \alpha}|\xi|^{1-2 \alpha}\left(\int_{0}^{t} e^{-(t-u) 4 \pi^{2} \xi^{2}} d B_{u}\right. & \\
& \left.+\left(e^{-4 \pi^{2} \xi^{2} t}-1\right) X_{0}(\xi)\right),
\end{aligned}
$$

with $X_{0}(\xi)$ a centered gaussian process with covariance such that $X(\xi, t)$ be stationary (with respect to $t$ ).

\subsection{Simulation of sample-Paths}

The last two representations can be used for simulating sample-paths of the fractional Brownian motion. Using (2.1), we have (see [10]) :

Proposition 2.1. Let $\pi^{n}$ be an increasing sequence of partitions of $[0,1]$ such that the mesh $\left|\pi^{n}\right|$ of $\pi^{n}$ tends to 0 as $n$ goes to infinity. The sequence of processes $\left\{W^{n}, n \geq 0\right\}$ defined by

$$
W_{t}^{n}=\sum_{t_{i}^{n} \in \pi^{n}} \frac{1}{t_{i+1}^{n}-t_{i}^{n}} \int_{t_{i}^{n}}^{t_{i+1}^{n}} K_{H}(t, s) d s\left(W_{t_{i+1}^{n}}^{1 / 2}-W_{t_{i}^{n}}^{1 / 2}\right)
$$

converges to $W^{H}$ in $L^{2}\left(\mathbf{P}_{1 / 2} \otimes d s\right)$. 
For $H>1 / 2$, one can consider the $W=\mathcal{C}([0,1], \mathbf{R})$-valued process

$$
\chi_{t} \stackrel{\text { def }}{=} \int_{0}^{t} K_{H}(., s) d B_{s}
$$

which is related to $W^{H}$ by the identity $\chi_{t}(t)=W_{t}^{H}$. Hence, expand $K_{H}(., s)$ on a basis $\left(e_{n}, n \in \mathbf{N}\right)$ of $W$, i.e. find $\left(\alpha_{n}(s), n \in \mathbf{N}\right)$ such that

$$
K_{H}(., s)=\lim _{n \rightarrow \infty} \sum_{j=0}^{n} \alpha_{j}(s) e_{j}(.)
$$

and compute $\sum_{j=0}^{n} \int_{0}^{t} \alpha_{j}(s) d B_{s} e_{j}(t)$ to obtain an approximation of $W_{t}^{H}$. By martingale inequalities, the precision of this method is directly related to the bounds we have on the approximation of $K_{H}(., s)$.

Using (2.3), the principle is to draw a sample-path of $X(\xi, t)$ by solving the stochastic differential equation it satisfies, then approximate the integral on the real line by well-chosen Riemann sums. Precise development of this approach and convergence results are given in [4].
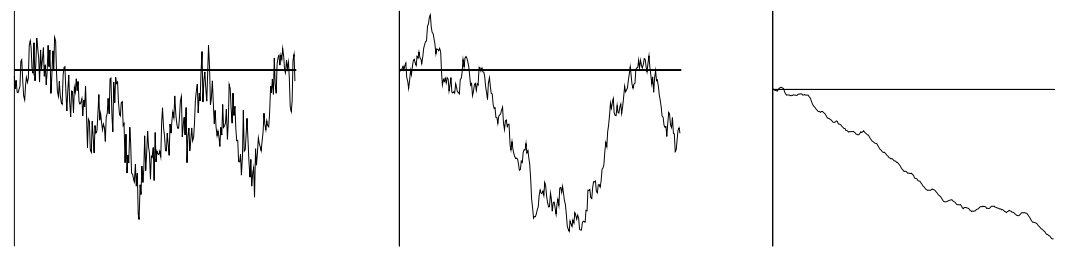

Figure 2. Typical sample-path for $H=0.2, H=0.5, H=0.8$.

\section{Properties}

The sample-paths of $W^{H}$ are almost-surely Hölder continuous of any order less than $H$, hence continuous but nowhere differentiable. The process $W^{H}$ has stationary increments and is self-similar :

$$
\left\{W_{\alpha t}^{H}, t \in[0, T]\right\} \stackrel{(d)}{=}\left\{\alpha^{H} W_{t}^{H}, t \in[0, T]\right\} .
$$

The increments are independent only when $H=\frac{1}{2}$, for $H>\frac{1}{2}$, the increments are positively correlated, and for $H<\frac{1}{2}$ they are negatively correlated (see [21]). As a consequence of the results in [1], we have

$$
\mathbf{P}_{H}\left(\limsup _{u \rightarrow 0^{+}} \frac{W_{u}^{H}}{u^{H} \sqrt{\log \log u^{-1}}}=\sqrt{V_{H}}\right)=1 .
$$

For $H<1 / 2$ the quadratic variation of $W^{H}$ is infinite whereas for $H>1 / 2$ it is null, hence in this latter case $W^{H}$ is a Dirichlet process. Meanwhile, for any $H$, the $1 / H$ variation is bounded, i.e.,

$$
0<\sup _{\pi} \mathbf{E}\left[\sum_{t_{i} \in \pi}\left|W_{t_{i+1}}-W_{t_{i}}\right|^{1 / H}\right]<+\infty .
$$

For $H<1 / 2$ there exists a bicontinuous local time (see [7]) and for $H>1 / 2$, the existence of a local time is ensured by the criterion of [15] but the joint continuity 
is an open problem. Using the standard Brownian motion defined in representation (2.1), one can easily show that

$$
\mathbf{E}\left[W_{t}^{H} \mid \mathcal{F}_{s}\right]=\int_{0}^{s} K_{H}(t, r) d B_{s} .
$$

Since the $\sigma$-fields generated respectively by $W^{H}$ and $B$ are the same, the ClarkOcone holds (for a more general result, see [11]) :

Proposition 3.1. For any $F \in L^{2}$, there exists $u$ adapted and square integrable such that

$$
F=\mathbf{E}[F]+\int_{0}^{1} u_{s} d B_{s} .
$$

The Reproducing Kernel Hilbert Space (or Cameron-Martin space) $\mathcal{H}_{H}$ of the fractional Brownian motion is described as

$$
\mathcal{H}_{H}=\left\{h: \exists \dot{h} \in L^{2}([0,1] ; \mathbf{R}) \text { such that } h(t)=\int_{0}^{t} K_{H}(t, s) \dot{h}(s) d s\right\}
$$

and the norm on $\mathcal{H}_{H}$ is given by $\|h\|_{\mathcal{H}_{H}}=\|\dot{h}\|_{L^{2}}$. The last but not the least standard result is the so-called Girsanov theorem :

Theorem 3.2 (Girsanov theorem). Let $u$ be a square integrable, adapted, process such that $\mathbf{E}\left[\Lambda_{1}^{u}\right]=1$, where

$$
\Lambda_{t}^{u} \stackrel{\text { def }}{=} \exp \left(\int_{0}^{t} u_{s} d B_{s}-1 / 2 \int_{0}^{t} u_{s}^{2} d s\right) .
$$

Let $\mathrm{Q}$ be the probability defined by

$$
\left.\frac{d \mathbf{Q}}{d \mathbf{P}_{H}}\right|_{\mathcal{F}_{t}^{H}}=\Lambda_{t}^{u} .
$$

The law of the process $\left\{W_{t}^{H}-\int_{0}^{t} K_{H}(t, s) u_{s} d s, t \in[0,1]\right\}$ under $\mathbf{Q}$ is the same as the law of $W^{H}$ under $\mathbf{P}_{H}$. In other words, for any $v$ adapted and square integrable, the law of the process

$$
\left\{\int_{0}^{t} K_{H}(t, s) v_{s} d B_{s}-\int_{0}^{t} K_{H}(t, s) u_{s} v_{s} d s, t \in[0,1]\right\} \text { under } \mathbf{Q}
$$

is the same as the law of the process

$$
\left\{\int_{0}^{t} K_{H}(t, s) v_{s} d B_{s}, t \in[0,1]\right\} \text { under } \mathbf{P}_{H} .
$$

\section{Stochastic calculus}

To model systems governed by a fractional Brownian motion, one is tempted to write

$$
d X_{t}=b\left(t, X_{t}\right) d t+\sigma\left(t, X_{t}\right) d W_{t}^{H}
$$

or equivalently

$$
X_{t}=X_{0}+\int_{0}^{t} b\left(s, X_{s}\right) d s+\int_{0}^{t} b\left(s, X_{s}\right) d W_{s}^{H} .
$$

However the meaning of the stochastic integral needs to be defined. Even more, if such an integral could be defined, this definition should permit us to be able to construct a stochastic calculus. If one mimics the Ito approach for the standard Brownian motion, the natural definition is :

$$
\int_{0}^{t} u_{s} d W_{s}^{H}=\lim _{|\pi| \rightarrow 0} \sum_{t_{i} \in \pi} u_{t_{i}}\left(W_{t_{i+1}}^{H}-W_{t_{i}}^{H}\right),
$$


where $\pi$ is a subdivision of $[0, t]$ and $|\pi|$ is its mesh. This approach has been followed by several authors with the restrictive assumption $H>1 / 2$ :

- Since for $H>1 / 2$ the $\mathrm{fBm}$ is a Dirichlet process, (4.1) can be given a sense using the approach developed by Föllmer in [14].

- Since the fBm has $1 / H$ bounded variation, one can use the work of Bertoin [3] in which it is proved that (4.1) converges whenever $u$ has $1 / \beta$-bounded variation with $\beta+H>1$ and $\beta \geq 2$. In the same vein, one can also cite the papers $[9,19]$ which consider more specifically the case of the fractional Brownian motion. Young (see [31]) and more recently Lyons [20] have extended these results so that there are now applicable to the fractional Brownian motion of Hurst parameter less than $1 / 2$.

- Using the Hölder continuity of the sample-paths of $W^{H}$, when $H>1 / 2$, Feyel and de la Pradelle [13] also prove that (4.1) converges when $u$ is $\beta$-Hölder continuous with $\beta+H>1$ (see [13]).

- One could also mention the works of Ciesielski and al. [5] and Zähle [32], where a path wise stochastic integral is defined through the notion of Besov space.

However, this approach is not really practical. As seen above, the class of processes $u$ such that (4.1) converges is far from being satisfactory. Constructing a stochastic calculus with this class using the above definition is almost impossible. For instance, for non-deterministic $u$, the expectation of $\int_{0}^{t} u_{s} d W_{s}^{H}$, defined by (4.1), is not computable. In fact, it is shown in [11] that

$$
\int_{0}^{1} u_{s} d W_{s}^{H}=\int_{0}^{t} K_{H}^{*}\left(u^{\prime}\right)(s) d B_{s}+\operatorname{trace}\left(\nabla K_{H}^{*}\left(u^{\prime}\right)\right),
$$

where $u^{\prime}$ is the derivative in the sense of distributions of $u$. Equation (4.2) means that the expectation of $\int_{0}^{t} u_{s} d W_{s}^{H}$ is strongly related to the expectation of the Gross-Sobolev derivative of $u$ and this is rarely known or even computable.

A second way of defining the stochastic integral may be the use of the Wiener integral associated to the $\mathrm{fBm}$ which is a Gaussian process. For any $H \in(0,1)$, the Wiener integral with respect to the fractional Brownian motion is usually defined as the linear extension from $\mathcal{H}_{H}$ in $L^{2}\left(\mathbf{P}_{H}\right)$ of the isometric map :

$$
d_{w}: R_{H}\left(t_{i}, .\right) \longmapsto W_{t_{i}} .
$$

Since $K_{H}\left(L^{2}([0,1])\right)=\mathcal{H}_{H}$, one could also define a Wiener integral for deterministic integrands belonging to $L^{2}([0,1])$ by considering the linear extension from $L^{2}([0,1])$ to $L^{2}\left(\mathbf{P}_{H}\right)$ of the isometry :

$$
\partial_{w}: K_{H}\left(t_{i}, .\right) \longmapsto W_{t_{i}} .
$$

When $H=1 / 2, K_{1 / 2}(t,)=.\mathbf{1}_{[0, t]}$ hence we have :

$$
\partial_{w}(h)=\lim _{n \rightarrow+\infty} \sum_{i=0}^{2^{n}-1} h_{i 2^{-n}}\left(W_{(i+1) 2^{-n}}-W_{i 2^{-n}}\right),
$$

for any continuous $h$. From (4.3), it is clear that (4.4) does not hold any more when $H \neq 1 / 2$. On the one hand, for $\alpha_{1}, \ldots, \alpha_{i}$ deterministic, we have

$$
\partial_{w}\left(\sum \alpha_{i}\left(K_{H}\left(t_{i+1}, .\right)-K_{H}\left(t_{i}, .\right)\right)\right)=\sum \alpha_{i}\left(W_{t_{i+1}}^{H}-W_{t_{i}}^{H}\right)
$$

and on the other hand, for the same $\alpha$ 's, we have

$$
\partial_{w}\left(\sum \alpha_{i}\left(\mathbf{1}_{\left[0, t_{i+1}\right]}-\mathbf{1}_{\left[0, t_{i}\right]}\right)\right)=\sum \alpha_{i}\left(B_{t_{i+1}}-B_{t_{i}}\right),
$$


where $B$ is still the standard Brownian linked to $W^{H}$ by (2.1). Consequently, to obtain the intuitive Riemann sums as in (4.1), one has to choose the step functions of the form

$$
\sum \alpha_{i}\left(K_{H}\left(t_{i+1}, .\right)-K_{H}\left(t_{i}, .\right)\right) .
$$

Unfortunately, it seems rather difficult to express a given function as a limit of such step functions since for $n$ fixed, the family $\left(K_{H}\left((i+1) 2^{-n},.\right)-K_{H}\left(i 2^{-n},.\right), i=\right.$ $\left.0 \ldots 2^{n}-1\right)$ is neither orthogonal nor simply orthogonalizable. The divergence, in the sense of the calculus of variations (see [30] for an account on stochastic calculus of variation), is an extension of the Wiener integral and this justifies our choice of using it as a stochastic integral with respect to the fractional Brownian motion. It is of no use for the purposes of this paper to go further into the depths of the Malliavin calculus. At present, what is really needed is the notion of anticipating (or Skohorod) integral with respect to the usual standard Brownian motion (see the appendix for a brief introduction). We denote it by $\delta_{H} B$. In what follows, $u$ denote an adapted and square integrable process. We define

$$
\int_{0}^{1} u_{s} d W_{s}=\int_{0}^{1} \mathcal{K}_{H}^{*} u(s) \delta_{H} W_{H}(s)
$$

where $\mathcal{K}_{H}=K_{1 / 2}^{-1} K_{H}$. When $H>1 / 2, \mathcal{K}_{H}$ is well defined since $\mathcal{H}_{H}$ is included in $\mathcal{H}_{1 / 2}$. For $H<1 / 2, \mathcal{K}_{H}$ can be defined by density since $\mathcal{H}_{1 / 2}$ is dense in $\mathcal{H}_{H}$. When $H>1 / 2$ we have the following identity provided that $u$ is deterministic and both sides exist :

$$
\int_{0}^{1} u_{s} \stackrel{0}{d} W_{s}=\lim _{\left|\pi_{n}\right| \rightarrow 0} \sum_{t_{i}^{n} \in \pi_{n}} u_{t_{i}^{n}}\left(W_{t_{i+1}^{n}}-W_{t_{i}^{n}}\right) .
$$

When $u \in \mathbb{D}_{2,1}^{H}\left(L^{2}([0,1])\right)$ and $\operatorname{trace}\left(\nabla K_{H} \mathcal{K}_{H}^{*} u\right)$ is well defined, we have :

$$
\int_{0}^{1} u_{s} \stackrel{o}{d} W_{s}=\lim _{\left|\pi_{n}\right| \rightarrow 0} \sum_{t_{i}^{n} \in \pi_{n}} u_{t_{i}^{n}}\left(W_{t_{i+1}^{n}}-W_{t_{i}^{n}}\right)-\operatorname{trace}\left(\nabla K_{H} \mathcal{K}_{H}^{*} u\right) .
$$

All this theory can be used to prove an Itô formula for $H>1 / 2$ and $f$ twice differentiable with bounded derivative.

$$
\begin{gathered}
f\left(W_{t}^{H}\right)-f(0)=H V_{H} \int_{0}^{t} f^{\prime \prime}\left(W_{s}^{H}\right) s^{2 H-1} d s \\
+\int_{0}^{t} s^{1 / 2-H} \int_{s}^{1} u^{H-1 / 2}(u-s)^{H-3 / 2} f^{\prime}\left(W_{u}^{H}\right) d u \delta_{H} W_{H}(s) . \\
\quad=H V_{H} \int_{0}^{t} f^{\prime \prime}\left(W_{s}^{H}\right) s^{2 H-1} d s+\int_{0}^{t} f^{\prime}\left(W_{s}^{H}\right) d W_{s}^{H} .
\end{gathered}
$$

This formula shows that whichever definition we choose for the stochastic integral with respect to the fractional Brownian motion, we will be forced to use anticipating calculus.

\section{Applications}

\subsection{Filtering theory}

Assume that we are given the observation $Y$ of a signal $X$ corrupted by a fractional Brownian motion noise. Assume furthermore that the original signal $X$, satisfies an evolution equation involving an other fbm possibly with a different Hurst index. The filtering problem consists of finding the conditional law of $X$ with respect to $Y$. The problem in its whole generality has been addressed in [8]. The usual strategy can be replicated here with some additional technical difficulties 
due to the lack of martingale properties. In order to show the main ideas, we will explicitly handle the following simple case :

$$
\begin{aligned}
& X_{t}=W_{t}^{1} \\
& Y_{t}=\int_{0}^{t} K_{2}(t, s) h\left(X_{s}\right) d s+W_{t}^{2},
\end{aligned}
$$

where $W^{1}$ and $W^{2}$ are two independent fractional Brownian motion of respective Hurst index $H_{1}$ and $H_{2}$. With $W^{i}$, is associated a standard Brownian motion $B^{i}$ by (2.1).

TheOREM 5.1. Let $\mathbf{Q}$ be the probability measure defined by

$$
\left.\frac{d \mathbf{Q}}{d \mathbf{P}_{H}}\right|_{\mathcal{F}_{t}} \stackrel{\text { def }}{=} \mathbf{E}\left(\Lambda_{T}^{h} \mid \mathcal{F}_{t}\right) \stackrel{\text { not }}{=} \Lambda_{t}^{h}
$$

where

$$
\Lambda_{t}^{h}=\exp \left[\int_{0}^{t} h\left(X_{s}\right) d B_{s}^{2}-\frac{1}{2} \int_{0}^{t} h\left(X_{s}\right)^{2} d s\right] .
$$

Under $\mathbf{Q}$, the processes:

$$
\tilde{W}_{t}^{1}=W_{t}^{1} \text { and } \tilde{W}_{t}^{2}=W_{t}^{2}-\alpha \int_{0}^{t} K_{2}(t, s) h\left(X_{s}\right) d s,
$$

are two independent fractional Brownian motion of Hurst index $\mathrm{H}_{1}$ and $\mathrm{H}_{2}$.

We give the proof to emphasize again the role of the infinite dimension.

Proof. For any $\left(t_{1}, \ldots, t_{n}\right) \in[0,1]^{n}$, consider the $2 n$ dimensional $\mathbf{P}_{H}$ martingale

$$
Z_{r}^{t_{1}, \ldots, t_{n}}=\left(\int_{0}^{r} K_{H_{1}}\left(t_{j}, s\right) d B_{s}^{1}, \int_{0}^{r} K_{H_{2}}\left(t_{j}, s\right) d B_{s}^{2}\right)_{j=1, \ldots, n}
$$

and the bounded variation process

$$
A_{r}^{t_{1}, \ldots, t_{n}}=\left(0, \int_{0}^{r} K_{H_{2}}\left(t_{j}, s\right) h\left(X_{s}\right) d s\right)_{j=1, \ldots, n} .
$$

The classical Girsanov theorem stands that for any bounded $f$,

$$
\left.\mathbf{E}_{\mathbf{Q}}\left[f\left(Z_{r}^{t_{1}, \ldots, t_{n}}-A_{r}^{t_{1}, \ldots, t_{n}}\right)\right]=\mathbf{E}\left[f\left(Z_{r}^{t_{1}, \ldots, t_{n}}\right)\right)\right] .
$$

Take $r=\sup _{i} t_{i}$ yields to

$$
\mathbf{E}_{\mathbf{Q}}\left[f\left(\tilde{W}_{t_{j}}^{i}, i=1,2,1 \leq j \leq n\right)\right]=\mathbf{E}\left[f\left(W_{t_{j}}^{i}, i=1,2,1 \leq j \leq n\right)\right]
$$

Hence the finite dimensional Q-laws of $\left(\tilde{W}^{1}, \tilde{W}^{2}\right)$ coincide with those of $\left(W^{1}, W^{2}\right)$ under $\mathbf{P}_{H}$.

The same reasoning shows that under $\mathbf{Q}$, the couple $(X, Y)$ has the same law as $\left(\tilde{W}^{1}, \tilde{W}^{2}\right)$. From here, it is classical to apply the Itô formula (provided that it exists hence we need to assume here that $\left.H_{1}>1 / 2\right)$ to the product $f\left(X_{t}\right) \Lambda_{t}^{h}$ and then compute the conditional expectations to obtain the so-called unnormalized filter $\sigma_{t}(f) \stackrel{\text { not }}{=} \mathbf{E}_{\mathbf{Q}}\left[f\left(X_{t}\right) \Lambda_{t}^{h} \mid \mathcal{Y}_{t}\right]$. The moral of the part of this work is that all the technical difficulties can be overcome but the final result is not satisfactory. Actually, one finds (see [8]) that the equation satisfied by $\sigma_{t}(f)$ is of the form :

$$
d \sigma_{t}(f)=\sigma_{t}\left(f^{\prime \prime} \psi\left(X_{t]}\right)\right) d t+\sigma_{t}(f . h) d B_{t}^{2}
$$

where $\psi\left(X_{t]}\right)$ is a measurable function of the whole sample-path of $X$ up to time $t$. This difficulty can be seen as a consequence of the long-range dependence of the fractional Brownian motion $W^{1}$. To overcome it, we filter the infinite dimensional 
process $\chi$. Using the Itô formula of Kuo [17] for Banach valued processes, for sufficiently regular $F: \mathbb{W} \rightarrow \mathbf{R}$ where $\mathbb{W}=\mathcal{C}_{0}([0,1] ; \mathbf{R})$, we find that the unnormalized filter $\tilde{\sigma}_{t}(F) \stackrel{\text { def }}{=} \mathbf{E}_{\mathbf{Q}}\left[F\left(\chi_{t}\right) \Lambda_{t}^{h} \mid \mathcal{Y}_{t}\right]$ solves the differential equation :

$$
\begin{aligned}
\tilde{\sigma}_{t}(F)=F\left(x_{0}\right)+\int_{0}^{t} \tilde{\sigma}_{s}\left(F . h \circ p_{s}\right) d B_{s}^{2} & \\
& +\frac{1}{2} \int_{0}^{t} \tilde{\sigma}_{s}\left(\left(K_{H_{i}}(., s)\right)^{*} D_{\alpha}^{2} F(.)\left(K_{H_{i}}(., s)\right)\right) d s,
\end{aligned}
$$

where $p_{s}(x)=x(s)$ for any $x \in \mathbb{W}$ and $D_{\alpha}^{2} F$ is the second order Fréchet dérivative of $F$ in the direction $\mathcal{I}_{\alpha, 2}$. As an example of the efficiency of this method, for $h=\mathrm{Id}$, choose $F(x)=\exp (i \beta x(1))$ where $x(1)$ is the value at time 1 of the element $x$ of $\mathbb{W}$, and set $\mathfrak{X}(t, \beta)=\tilde{\sigma}_{t}(F)$, we obtain the following linear partial stochastic differential equation :

$$
\begin{aligned}
d \mathfrak{X}(t, \beta) & =-\beta^{2} K_{H}(1, t)^{2} \mathfrak{X}(t, \beta) d t-i \frac{\partial \mathfrak{X}}{\partial \beta}(t, \beta) d B_{t}^{2}, \\
\mathfrak{X}(0, \beta) & =x_{0} .
\end{aligned}
$$

It is then tricky but possible to find an explicit Gaussian solution of this equation and thus to obtain the Kalman-Bucy filter of $X$ with respect to $Y$ (see [16] for a related result in which $W^{2}$ is a standard Brownian motion).

\subsection{Queuing NeTWORKS}

The importance of fractional Brownian motion for dimensioning buffers in telecommunications networks has been first stressed by Norros in [25]. Since then, numerous studies have been done on the subject (cf. [26, 12, 2]). Actually, the framework is the following : consider a single-server queue with constant service rate (taken to be $c$ unit of work per unit of time) fed by a process $\left\{X_{t}, t \geq 0\right\}$, the workload process is denoted by $\left\{W_{t}, t \geq 0\right\}$ and $W$ is the workload at stationary regime. When $X_{t}=m t+\sqrt{a m} W_{t}^{H}$, it is known from [25] that the buffer requirements, to keep a fixed loss probability, increase dramatically when $H$ is close to 1 . Namely, this is due to the fact that, for $x$ large,

$$
P(W>x) \simeq \exp \left(-\frac{(c-m)^{2 H}}{2 \kappa(H)^{2} a m} x^{2-2 H}\right)
$$

where $\kappa(H)=H^{H}(1-H)^{1-H}$. More generally, when $X$ is the solution of the following equation

$$
X_{t}=\sum_{i=1}^{n} \int_{0}^{t} K_{H_{i}}(t, s) b_{i}\left(s, X_{s}\right) d s+\int_{0}^{t} K_{H_{i}}(t, s) \sigma_{i}\left(s, X_{s}\right) \delta_{H_{i}} B_{s}^{i}
$$

where $\left(B^{i}, i \in 1, \ldots, n\right)$ is a collection of independent standard Brownian motion, a similar result holds with $H=\sup _{1 \leq i \leq n} H_{i}$, namely we have :

$$
\lim _{x \rightarrow \infty} x^{2 H-2} \ln P(W \geq x\}=-\frac{1}{2 V_{H}} \frac{c^{2 H}}{\kappa(H)^{2}} .
$$

\subsection{Mathematical finance}

In mathematical finance, the main problem is the pricing of finance products. By a call option of an asset $S$ and expiration time $T$, it is meant a contract between two partners $A$ and $B$, by the virtue of which $B$ can buy a share of $S$ at price $K$ at time $T$. If at time $T$, the price of the asset is smaller than $K$ then $A$ losses money whereas if the price of the asset is greater than $K, B$ is not constrained to buy any share (and in fact won't buy any). The value of $K$ is fixed by the market and thus 
the problem is to determine the price $C, B$ should pay now to $A$ for this contract. The celebrated Black-Scholes formula is based on the assumption that the asset price satisfies the following time evolution :

$$
d S_{t}=\mu S_{t} d t+\sigma S_{t} d B_{t} \text {, i.e., } S_{t}=S_{0} \exp \mu t+\sigma B_{t},
$$

where $\mu$ and $\sigma$ are constant. The question now is thus to know what happens when $B$ is replaced by an $\mathrm{fBm}(\mathrm{H})$. In fact, in this situation, Rogers [27] and more recently Shirayev shown that there exists some arbitrage opportunity, i.e., one can earn money from a null initial fortune. This represents a strong limitation for this model, a possible workaround which remains to be examined in detail is to change the kernel $K_{H}(t, s)$ into a kernel smoother with respect to $s$ in the neighborhood of $s=0$.

\section{Appendix A. Deterministic fractional Calculus}

For $f \in \mathcal{L}^{1}([0,1])$, the left and right fractional integrals of $f$ are defined by :

$$
\begin{aligned}
& \left(I_{0}^{\alpha} f\right)(x) \stackrel{\text { def }}{=} \frac{1}{\Gamma(\alpha)} \int_{0}^{x} f(t)(x-t)^{\alpha-1} d t, x \geq a, \\
& \left(I_{1}^{\alpha} f\right)(x) \stackrel{\text { def }}{=} \frac{1}{\Gamma(\alpha)} \int_{x}^{1} f(t)(t-x)^{\alpha-1} d t, x \leq b,
\end{aligned}
$$

where $\alpha>0$ and $I^{0}=\mathrm{Id}$. For any $\alpha \geq 0$, any $f \in \mathcal{L}^{p}([0,1])$ and $g \in \mathcal{L}^{q}([0,1])$ where $p^{-1}+q^{-1} \leq \alpha$, we have :

$$
\int_{0}^{1} f(s)\left(I_{0}^{\alpha}+g\right)(s) d s=\int_{0}^{1}\left(I_{1}^{\alpha}-f\right)(s) g(s) d s .
$$

The Besov space $I_{0^{+}}^{\alpha}\left(\mathcal{L}^{p}\right) \stackrel{\text { not }}{=} \mathcal{I}_{\alpha, p}$ is usually equipped with the norm :

$$
\|f\|_{\mathcal{I}_{\alpha, p}}=\left\|I_{0+}^{-\alpha} f\right\|_{\mathcal{L}^{p}} .
$$

We then have the following continuity results (see [13, 28]) :

Proposition A.1.

i. If $0<\alpha<1,1<p<1 / \alpha$, then $I_{0+}^{\alpha}$ is a bounded operator from $\mathcal{L}^{p}([0,1])$ into $\mathcal{L}^{q}([0,1])$ with $q=p(1-\alpha p)^{-1}$.

ii. For any $0<\alpha<1$ and any $p \geq 1, \mathcal{I}_{\alpha, p}$ is continuously embedded in $\operatorname{Hol}(\alpha-1 / p)$ provided that $\alpha-1 / p>0$. Hol $(\nu)$ denotes the space of Hölder-continuous functions, null at time 0, equipped with the usual norm.

iii. For any $0<\alpha<\beta<1, \operatorname{Hol}(\beta)$ is compactly embedded in $\mathcal{I}_{\alpha, \infty}$.

iv. Let $0<\alpha<1, \varphi \in \mathcal{L}^{p}$ for some $p>1$ and $\mu>-1+1 / p$. There exists $\phi \in \mathcal{L}^{p}$ such that

$$
I_{0^{+}}^{\alpha}\left(s^{\mu} \varphi\right)(t)=t^{\mu} I_{0^{+}}^{\alpha}(\phi)(t) \text { and }\|\phi\|_{\mathcal{L}^{p}} \leq c\|\varphi\|_{\mathcal{L}^{p}} .
$$

By $I_{0^{+}}^{-\alpha}$, respectively $I_{1^{-}}^{-\alpha}$, we mean the inverse map of $I_{0^{+}}^{\alpha}$, respectively $I_{1^{-}}^{\alpha}$.

\section{Appendix B. Anticipating stochastic calculus}

The definition of the so-called divergence and hence of our integral needs the definition of the Gross-Sobolev derivative.

Definition B.1. Let $X$ be a separable Hilbert space. For an $X$ valued smooth cylindric functional $F$ of the form, $F(\omega)=f\left(W_{t_{1}}^{H}, \ldots, W_{t_{n}}^{H}\right) x$, where $f$ belongs to 
the Schwartz space on $\mathbf{R}^{n}$ and $x$ to $X$, the Gross-Sobolev derivative of $\mathrm{F}$, denoted by $\nabla F$, is defined by :

$$
\nabla F(\omega)=\sum_{i=1}^{n} \frac{\partial f}{\partial x_{i}}\left(W_{t_{1}}^{H}, \ldots, W_{t_{n}}^{H}\right) R_{H}\left(t_{i}, .\right) \otimes x .
$$

For any $p \geq 1$ and any $k \geq 0$, the Sobolev space $\mathbb{D}_{p, k, H}(X)$ is the completion of the set $\mathcal{S}$ of cylindric functionals with respect to the norm :

$$
\|F\|_{p, k, H} \stackrel{\text { def }}{=}\|F\|_{L^{p}}+\left\|\left|\nabla^{(k)} F\right|_{H S}\right\|_{L^{p}}
$$

where ||$_{H S}$ stands for the Hilbert-Schmidt norm. For any $k<0, \mathbb{D}_{p, k, H}(X)$ is the strong dual of $\mathbb{D}_{p,-k, H}(X)$. The space $\mathbb{D}_{\infty}(X)=\cap_{p \geq 1, k>0} \mathbb{D}_{p, k, H}(X)$ is the space of test functions and the space $\mathbb{D}_{-\infty}(X)=\cup_{p \geq 1, k<0} \mathbb{D}_{p, k, H}(X)$ is the space of distributions. It is well known that $\nabla$ can be extended as a continuous operator from $\mathbb{D}_{p, k, H}$ into $\mathbb{D}_{p, k-1, H}\left(\mathcal{H}_{H}\right)$, for any $p \geq 1$ and any $k$. By $\dot{\nabla} F$, we mean the inverse image of $\nabla F$ by the map $K_{H}$. Moreover, for any once Gross-Sobolev differentiable random variable $\varphi$, for any $\psi \in L^{0}\left(\mathcal{H}_{H}\right)$, we set :

$$
\nabla_{\psi} \varphi \stackrel{\text { def }}{=}\langle\nabla \varphi, \psi\rangle_{\mathcal{H}_{H}}
$$

We say that a process $u$ belongs to $\operatorname{Dom}_{p} \delta_{H}$ when there exists $c$ such that for any $F \in \mathbb{D}_{p^{\star}, 1, H}:$

$$
\left|\mathbf{E}\left[\langle\nabla F, u\rangle_{\mathcal{H}_{H}}\right]\right| \leq c\|F\|_{L^{p^{\star}}(W)}
$$

and we define $\delta_{H} u$ by

$$
\mathbf{E}\left[\langle\nabla F, u\rangle_{\mathcal{H}_{H}}\right]=\mathbf{E}\left[F . \delta_{H} u\right] .
$$

Notation 1. Here and hereafter, we denote by $p^{\star}$ the conjugate of $p$, i.e., $1 / p^{\star}+$ $1 / p=1$.

$\delta_{H}$, called the divergence operator, is a continuous operator from $\mathbb{D}_{p, k, H}\left(\mathcal{H}_{H}\right)$ into $\mathbb{D}_{p, k-1, H}$. Since a divergence has to be applied to an element of the CameronMartin space and stochastic integral is usually defined for elements of $L^{2}([0,1])$, we introduce the (probably misleading) notation $\delta_{H} W_{H}(s)$ in the equality :

$$
\delta_{H}\left(K_{H} u\right)=\int u_{s} \delta_{H} W_{H}(s)
$$

where $u$ belongs to $L^{2}([0,1])$ and is such that $K_{H} u$ belongs to the domain of the divergence. It turns out (this is Theorem 4.8 of [11]) that this integral coincides with the stochastic integral with respect to a standard Brownian motion constructed on the space where the $\mathrm{fBm}$ is defined, that is to say, for $u$ adapted :

$$
\delta_{H}\left(K_{H} u\right)=\int u_{s} d B_{s} .
$$

\section{REFERENCES}

[1] M.A. Arcones, On the Law of the Iterated Logarithm for Gaussian Processes, Journal of Theoretical Probability 8 (1995), no. 4, 877-904.

[2] S. Belly and L. Decreusefond, Multi-dimensional Fractional Brownian Motion and some Applications to Queuing Theory, Fractals in Engineering (Lévy-Véhel and Tricot, eds.), 1997, pp. $170-185$.

[3] J. Bertoin, Sur une intégrale pour les processus à $\alpha$ variation bornée, The Annals of Probability 17 (1989), no. 4, 1521-1535.

[4] P. Carmona, L. Coutin, and G. Montseny, Applications of a Representation of Long-Memory Gaussian Processes, preprint, 1998.

[5] Z. Ciesielski, G. Kerkyacharian, and B. Roynette, Quelques espaces fonctionnels associés à des processus gaussiens, Studia Mathematica 107 (1993), no. 2, 171-204.

[6] R. Coelho and L. Decreusefond, Video Correlated Traffic Models for Broadband Communications Networks, Proceedings ot the ITC Seminar on Teletraffic management, 1995. 
[7] M. Cörgö, Z.Y. Lin, and Q.M. Shao, On Moduli of Continuity for Local Times of Gaussian Processes, Stochastic Processes and their Applications 58 (1995), 1-21.

[8] L. Coutin and L. Decreusefond, Non-linear Filtering Theory for Fractional Brownian Motion, Submitted, 1997.

[9] W. Dai and C.C. Heyde, Itô's Formula with respect to Fractional Brownian Motion and its Application, Journal of applied and stochastic analysis 9 (1996), 439-458.

[10] L. Decreusefond and N. Lavaud, Simulation of the fractional Brownian motion and application to the fluid queue. Proceedings of the ATNAC'96 conference, 1996.

[11] L. Decreusefond and A.S. Üstünel, Stochastic Analysis of the Fractional Brownian Motion, Potential Analysis (1998).

[12] N.G. Duffield and N. O'Connell, Large Deviations and Overflow Probabilities for the General Single Server Queue, with Applications, Proc. Cambridge Phil. Soc., 1995.

[13] D. Feyel and A. de La Pradelle, Fractional Integrals and Brownian Processes, Preprint, Université d'Évry, 1996, 1996.

[14] H. Föllmer, Calcul d'Itô sans probabibilité, Séminaire de probabilités, Springer-Verlag, 1980, pp. $143-150$

[15] D. Geman and J. Horowitz, Occupations Densities, The Annals of Probability 8 (1980), no. 1 , $1-67$.

[16] M.L. Kleptsyna, Kloeden P.E., and V.V. Anh, Linear flltering with fractional Brownian motion, Preprint, 1996.

[17] H.H. Kuo, Gaussian Measures in Banach Spaces, Lect. Notes in Mathematics, vol. 463, Springer-Verlag, 1975

[18] W.E Leland, M.S. Taqqu, W. Willinger, and D.V. Wilson, On the Self-similar Nature of Ethernet Traffic, IEEE/ACM trans. Networking 2 (1994), no. 1, 1-15.

[19] S.J. Lin, Stochastic analysis of fractional Brownian motions, To appear in Stochastics, 1996, 1996.

[20] T. Lyons, Differential equations driven by rough signals. I. An extension of an inequality of L. C. Young, Mathematical Research Letters 4 (1994), 451-464.

[21] B. Mandelbrot and J. Van Ness, Fractional Brownian Motions, Fractional Noises and Applications, SIAM Review 10 (1968), no. 4, 422-437.

[22] G. Montseny, J. Audounet, B. Mbodje, Optimal models of fractional integrators and application to systems with fading memory, IEEE SMC's conference, Le Touquet (France), 1993.

[23] E. Moulines and P. Soulier, Log-periodogram Regression of Time Series with Long Range Dependence, submitted to Annals of Statistics, 1996.

[24] A.F. Nikiforov and V.B. Uvarov, Special Functions of Mathematical Physics, Birkhäuser, 1988.

[25] I. Norros, A Storage Modelwith Self-Similar Inputs, Queuing Systems 16 (1994), 387-396.

[26] _ On the Use of the Fractional Brownian Motion in the Theory of Connectionless Networks, IEEE Jour. on Sel. Ar. in Communications 13 (1995), no. 6, 953-962.

[27] L.C. Rogers, Arbitrage with Fractional Brownian Motion, Mathematical Finance 7 (1997), no. $1,95-105$.

[28] S.G. Samko, A.A. Kilbas, and O.I. Marichev, Fractional Integrals and Derivatives, Gordon \& Breach Science, 1993

[29] M. Taqqu, V. Teverovsky, and W. Willinger, Estimators for Long-Range Dependence: An Empirical Study, Fractals 3 (1995), no. 4, 785-788.

[30] A.S. Üstünel, An Introduction to Analysis on Wiener Space, Lectures Notes in Mathematics, vol. 1610, Springer-Verlag, 1995.

[31] L.C. Young, An Inequality of Hölder Type, connected with Stieltjes Integration, Acta Math. 67 (1936), 251-282.

[32] M. Zähle, Integration with respect to Fractal Functions and Stochastic Calculus, I, Probability Theory and Related Fields 111, no. 3, 333-378. 